\title{
Radial Velocities with Gaia
}

\section{Mark Cropper ${ }^{1}$, David Katz ${ }^{2}$, Ulisse Munari ${ }^{3}$, Tomaz Zwitter ${ }^{4}$ and Andrew Holland ${ }^{5}$}

\author{
${ }^{1}$ Mullard Space Science Laboratory, University College London, Holmbury St Mary, Dorking, \\ Surrey RH56NT, UK email: msc@mssl.ucl.ac.uk \\ ${ }^{2}$ Observatoire de Paris, GEPI, 5 Place Jules Janssen, F-92195 Meudon, France \\ ${ }^{3}$ Osservatorio Astronomico di Padova INAF, sede di Asiago, 36012 Asiago (VI), Italy \\ ${ }^{4}$ University of Ljubljana, Department of Physics, Jadranska 19, 1000 Ljubljana, Slovenia \\ ${ }^{5}$ Centre for Electronic Imaging, School of Engineering and Design, Brunel University, \\ Uxbridge, UB8 3PH, UK
}

\begin{abstract}
Gaia is a mission to map the positions and determine the velocities of 1 billion stars in the Galaxy, in order to determine how it was formed and how it has evolved. Spectroscopy provides one component of the space velocities for 200 million objects, and, in addition, astrophysical information such as temperature and metallicity for the brighter 40 million stars in a large unbiased Galactic sample. Together the instruments on Gaia will transform our knowledge of the Galaxy. We describe here a sample of the science that will be done with Gaia concentrating on aspects in which the spectroscopy plays a central role.
\end{abstract}

\section{Introduction}

Gaia is an ESA Cornerstone science mission due for launch in 2011. Gaia's role is to determine how the Galaxy was born and how it has evolved, and it will do this by measuring the positions and space velocities of $\sim 10^{9}$ stars. The positions will be obtained by precise astrometry at the level of $10 \mu \operatorname{arcsec}$ (for stars of $V=15$ ), with the distances following though parallax measurements. Transverse space velocities are determined from proper motions throughout the six-year mission, while the radial velocities are obtained via a dedicated Radial Velocity Spectrometer (RVS) with a resolving power of 11500 and working in a band from $8480-8740 \AA$ around the Ca triplet. Gaia will also have a photometric capability, allowing luminosities to be determined, and, together with the RVS, will provide detailed physical characteristics (temperature, metallicity, gravity, etc.) of each object observed. It should be noted that owing to the scanning of the satellite, the observations are multi-epoch, providing information on variability and binarity. Astrometric and photometric measurements extend to beyond $V=20$, while spectroscopic measurements reach $V=17$. The completeness and scale of this dataset will be unprecedented, with selection functions that are clear and simply treated.

The scientific harvest of Gaia will be far-reaching, extending beyond the Galaxy to the local group, to the discovery of several supernovae and 250 QSOs per day, many of them lensed. It will find thousands of new Jupiter-mass planets, and within our Solar system, will detect large numbers of Kuiper-belt objects and asteroids. Gaia will impact profoundly on fundamental physics, for example providing tight constraints on the distribution and characteristics of Dark Matter, on the Modified Newtonian Dynamics (MOND) theories and on changes in the gravitational constant. An overview of the mission, including the science case, is available from Perryman et al. (2001), while up-to-date information can be obtained from the project website at http://www.rssd.esa.int/GAIA/. 

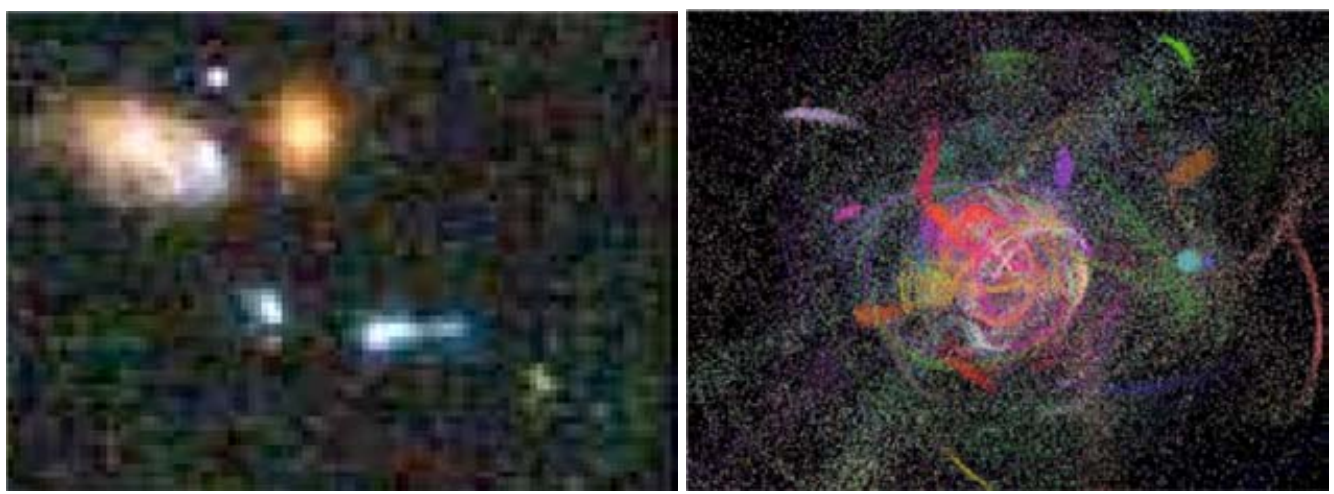

Figure 1. Left: Detail from the Hubble Space Telescope Ultra-Deep Field. Image courtesy of NASA. Right: A simulation depicting the formation of a halo by the accretion of 50 dwarf galaxies at various times during the last $10 \mathrm{Gyr}$. Image by Paul Harding, available from http://burro.astr.cwru.edu/heather/spag.html (Harding et al. 2001)

Gaia builds on the legacy of ESA's successful Hipparcos mission, which pioneered space-based astrometry. The scientific harvest from Hipparcos was diverse (see, for example, Perryman 1997). However, Hipparcos lacked a facility to measure the radial motions, with implications that were appreciated at the time. One example was due to Blaauw (1988):

"... there are, of course many things left that one would like to see done. One of them, perhaps the most urgent, is in the field of radial velocities. . . there is no really dedicated Hipparcos radial velocity programme... may we be sure that, when the Hipparcos proper motions become available, we will not be faced with a deplorable lack of data on the third component of stellar motions?"

Since then the absence of radial velocities for the Hipparcos data has been noted several times (e.g. Binney et al. 1997). While a large dataset of some 13500 velocities has recently become available (Nordström et al. 2004), it should be noted that this is still only $10 \%$ of the Hipparcos dataset, and the situation is expected to remain unsatisfactory. The Nordström et al. (2004) catalog contains F and G dwarf stars to $V \sim 10$ with the peak in the distribution at $V \sim 8.5$.

It was understood early on in the conceptual design of Gaia (Favata \& Perryman 1997) that, given the scale of the output catalog, the measurement of radial velocities would need to be obtained in parallel with the astrometric measurements. The mission was therefore designed with the RVS as an essential component of the instrument suite. This paper provides a brief overview of the science return from the RVS. More complete expositions can be obtained from Katz et al. (2004) and Wilkinson et al. (2004), with a wider view available from the Monte Rosa Conference proceedings (e.g. Munari 2003a).

\section{RVS Science Topics}

The highest priority science areas for RVS include the perspective acceleration correction (essential for astrometric measurements), thin disk, thick disk and halo kinematics, and the determination of the Galactic gravitational potential. Other science goals include mapping reddening, spiral arm and open cluster kinematics, binary systems, chemical abundance gradients, and the Galaxy disk warp.

RVS will achieve $<1 \mathrm{~km} \mathrm{~s}^{-1}$ velocity accuracy for $\sim 4 \times 10^{7}$ stars, and $<15 \mathrm{~km} \mathrm{~s}^{-1}$ accuracy for $\sim 10^{8}$ stars. 

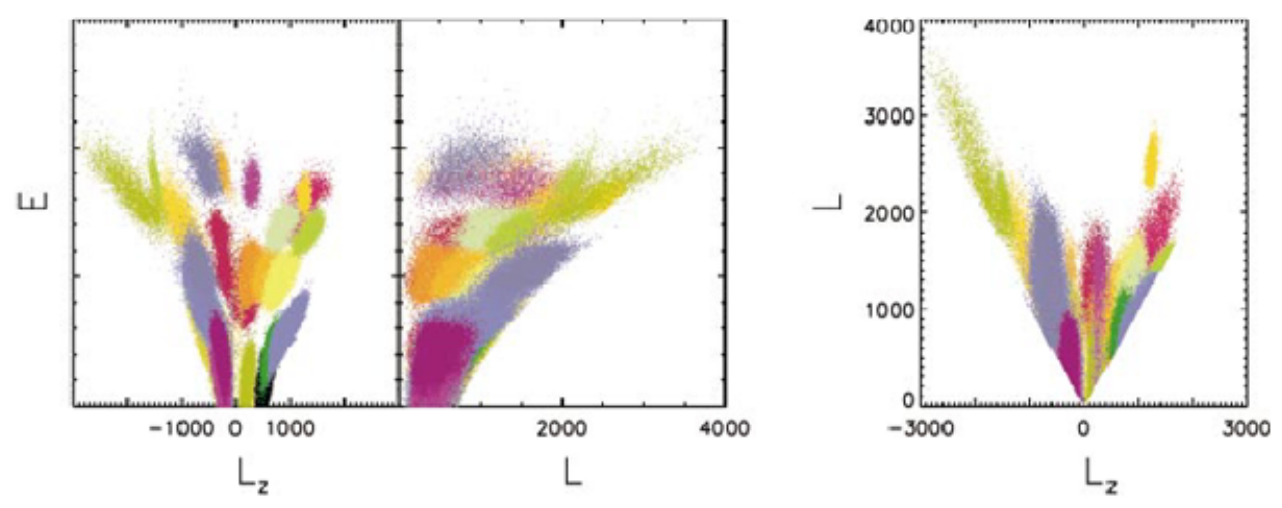

Figure 2. The distribution of particles within Galactic halo in the integrals of motion space after 12 Gyr. Particles denote bright giants $\left(M_{V}=1\right)$ with color coding representing different accreted satellites. From Helmi \& de Zeeuw (2000).

This section examines some of the areas of Gaia science in which spectroscopy plays a central role.

\subsection{Halo Streams}

The Milky Way is the only place where direct tests of galaxy formation scenarios can be made. Gaia's main quest is to understand how the Galaxy was formed and how it has evolved. This has been a long-standing issue in astronomy since the early seminal work in this area (such as Kapteyn 1922). For some time the accepted model was that of Eggen, Lynden-Bell \& Sandage (1962), in which the Galaxy formed in a monolithic collapse, with the halo as the oldest component, and the flattened disk resulting from conserved angular momentum as the youngest. More recent work has emphasised the importance of hierarchical merging of smaller systems with the Galaxy, perhaps responsible for generating the thick disk (Quinn, Hernquist \& Fullagar 1993). Recent images from the HST Deep Field and Ultra-Deep Field (Fig. 1, left) have shown how irregular most galaxies are at early epochs. This results (at least partly) from a combination of the relaxation of the dark matter halo and relatively active merging, together with the effects of early star formation activity.

Because of the long timescale for interactions of the halo stars, the best place to search for evidence of the merger history of the Galaxy is in the halo, since this is where relics would retain their identity longest. This led several groups to examine the possibilities from a theoretical point of view (Johnston, Hernquist \& Bolte 1996; Helmi \& White 1999). Fig. 1 (right) shows a simulation in which the outer halo is composed of a number of different streams, with many older streams widely spread throughout the halo. Helmi \& de Zeeuw (2000) addressed this issue from the point of view of the integrals of motion (energy, momentum) and found that these quantities for individual satellites retained their identity despite the spatial dispersion of their stars (see Fig. 2). This raised the possibility of tracing up to $80 \%$ of the merger history of the Galaxy (i.e. to $z<4$ ). At the same time progress was made observationally in identifying the disruption of satellites, for example in the dSgr galaxy where this process has proceeded towards almost complete disruption (Ibata, Gilmore \& Irwin 1994).

For this work to identify the various merger components in the merger scenarios, the integrals of motion (energy, momentum) must be derived from 6-dimensional position and velocity information. These motions are derived mainly from the kinematics of halo 


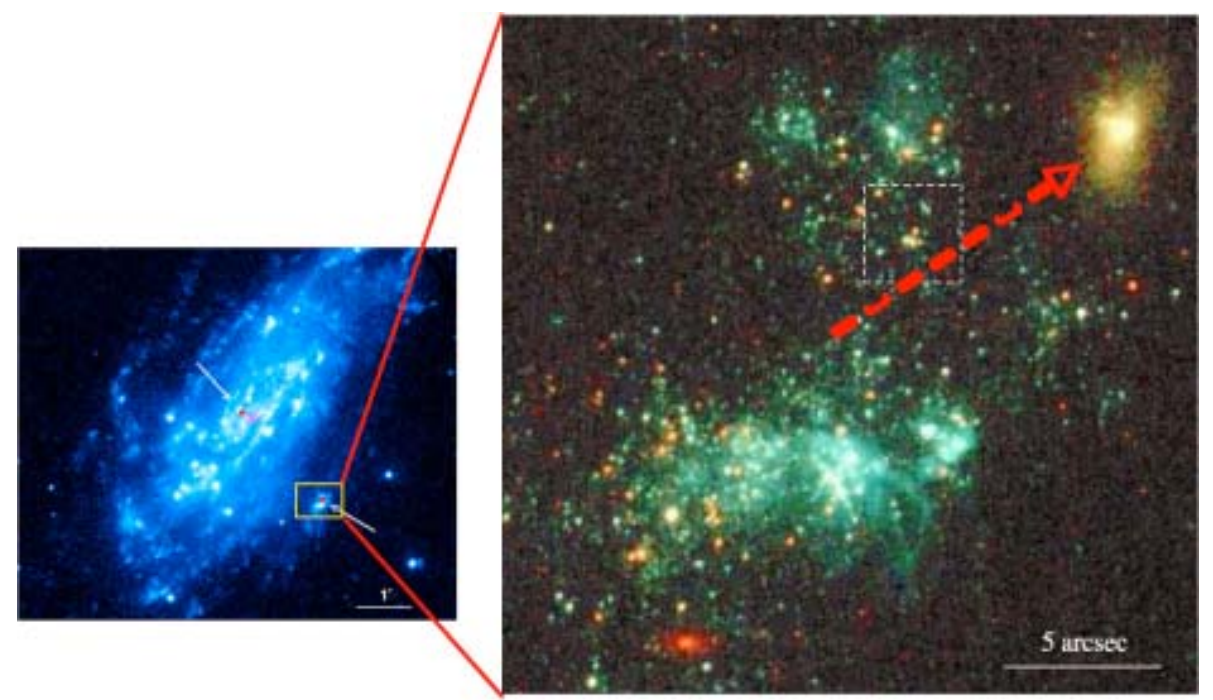

Figure 3. Star formation in the outskirts of NGC 4559. On the left is a UV image from the Optical Monitor on XMM-Newton while the enlarged image on the right is a 3-colour HST image. Soria et al. (2004) suggest this region is caused by the passing of a dwarf spheroidal galaxy (orange, upper right) through the outer disk of NGC 4558. From Soria et al. (2004).

stars with $V>16$, and it is necessary to reach $V>17$ to generate a sufficient sample. This is a significant driver for RVS performance.

\subsection{Solar System Origins and Evolution}

Despite the greater loss of information from kinematic motions within the disk compared to the halo arising from the greater number of interactions (mostly with spiral arms and molecular clouds), because of the accuracy and completeness of the astrometric and velocity measurements, Gaia will provide information on the origin of the Solar system and its subsequent evolution. It should be possible to distinguish among different scenarios, for example, whether the Sun formed within our main Galaxy, or whether it was incorporated as the result of a merger, as currently suggested for Arcturus (Navarro et al. 2004). Alternatively, because the extent to which star formation is triggered by mergers has become increasingly evident, for example in the Antennae galaxies, or NGC 4559 (Fig. 3), was the Solar system formed as a result of such a merger or interaction?

With regards to the more recent evolution of the Solar system and life within it, it was possible with Hipparcos data to reconstruct the motions of stars in the Solar vicinity over the past few Myr to examine which have passed sufficiently close to disturb the Oort Cloud, which is thought to extend a significant part of the distance to the nearest star (Algol passed within $2.5 \mathrm{pc}$ of the Sun $7 \mathrm{Myr}$ ago). For Gaia the full space motions will be known with much greater accuracy, so that ancient star encounters can be examined over geological timescales $>200 \mathrm{Myr}$. This will make it possible to test suggestions that extinctions in the number of species of life have been caused by cometary impacts resulting from disturbances of the Oort Cloud. It will also be possible to predict future nemeses.

\subsection{Normal Stars}

"Normal" stars, whether single or in resolved binaries, will form the bulk of the Gaia harvest. This will be a huge homogeneous sample for which astrophysical parameters 


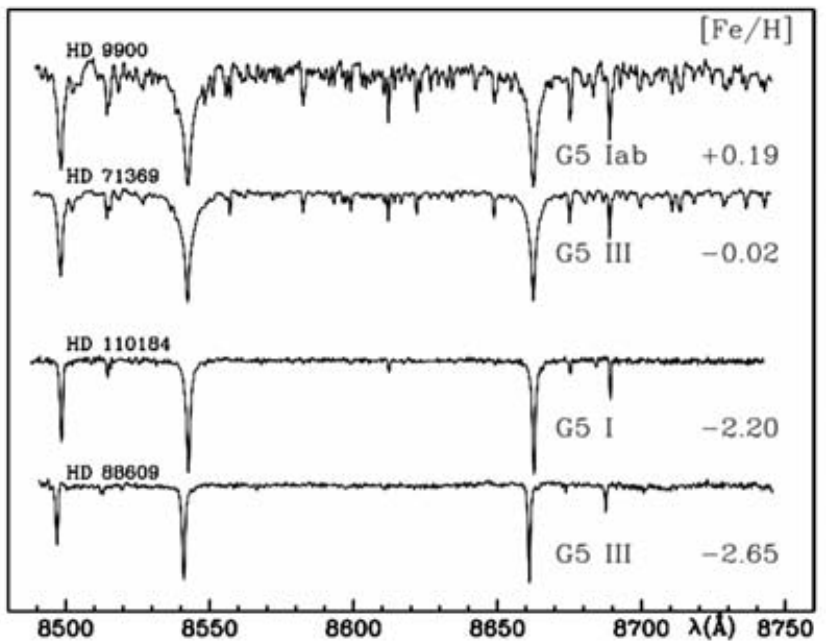

Figure 4. A metallicity sequence for spectra of G5 giant and supergiant stars. From Munari (2003a).

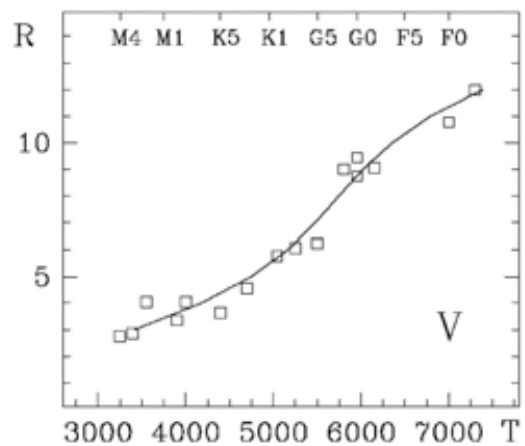

$$
\begin{aligned}
\mathrm{R}= & \frac{\text { CaII } 8542}{\text { FeI } 8688} \\
\mathrm{~T}= & -607.8+1876.0 \mathrm{R}+ \\
& -204.0 \mathrm{R}^{2}+8.6 \mathrm{R}^{3}
\end{aligned}
$$

Figure 5. A calibration of the ratio between Ca II $8542 \AA$ and Fe I $8688 \AA$ line equivalent widths for dwarf stars as a function of temperature. From Boschi et al. (2003)

such as effective temperature, metallicity, gravity and rotation can be measured. From these can be inferred the masses, luminosities and ages of the stars, and when combined with the kinematics, this will form the basis for the work on the chemical evolution of the Galaxy, on the IMF and star formation rates and on feedback processes. Being a spectrograph, RVS is an essential component in this, uniquely providing the detailed elemental abundances and rotations, and independent measures of $T_{\text {eff }}$ and gravity. An example is shown in Fig. 4 , where a sequence in metallicity $[\mathrm{Fe} / \mathrm{H}]$ in giants and supergiants is evident from the strength of the absorption lines of elements other than $\mathrm{Ca}$ (note that the Ca triplet is a strong spectral feature, and thus provides a good velocity marker even in extremely metal-poor stars). Fig. 5 shows an example calibration of the ratio of the CaII $8542 \AA$ and Fe I $8688 \AA$ lines with temperature.

Such detailed astrophysical information will be obtained for the brighter stars within the sample $V<15$. This is still a sample an order of magnitude larger than that available from any foreseen spectral surveys. 


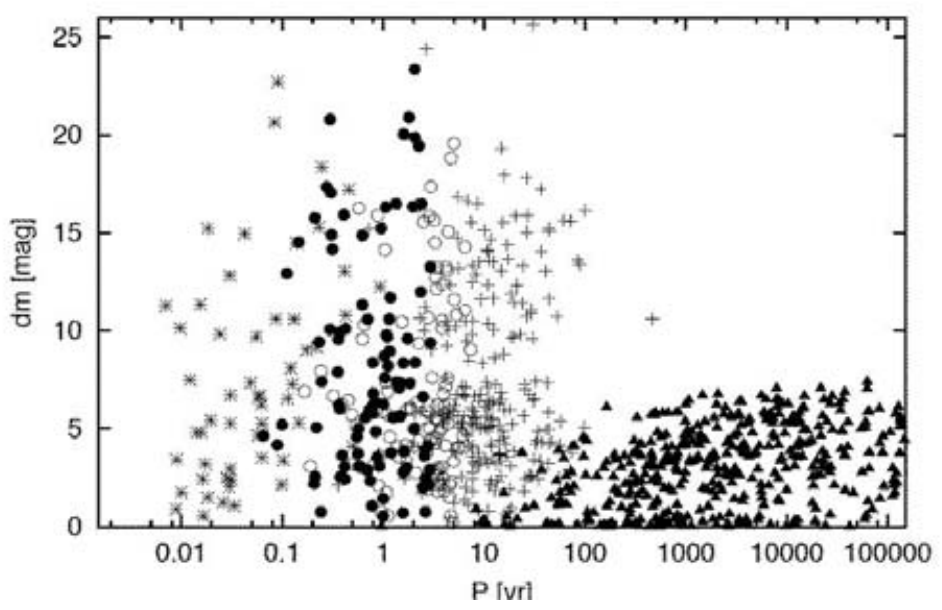

Figure 6. The properties for binaries as a function of period (years) and visual magnitude difference of the stellar components, for $V<15$. Each mark represents 5000 systems. Binaries detected by spectroscopy alone are designated by $*$, while those detected by both astrometry and spectroscopy are designated by $\bullet$. Other symbols indicate detection by astrometry alone. From Söderhjelm (2003).

\subsection{Binaries}

Perhaps up to $75 \%$ of stars in the Galaxy exist as binaries, but the real fraction is still not well known (e.g. Duchêne 1999). The spectrum of separations and mass ratios is also not well characterised, and is affected strongly by selection effects. Binaries are important because they are (except in exceptional circumstances) coeval pairs. They are thus direct tests of stellar evolution, and they offer the opportunity of direct mass and radius measurements, essential inputs to our understanding of stellar structure. Examining the binary population within different Galactic constituents (halo, thick disk, thin disk) can determine how stellar parameters depend on mass, age and chemical abundance. Moreover, a significant fraction of the angular momentum in the initial star forming cloud is locked up in binary and multiple systems, so it is impossible to examine the nature of star formation in any detail without including in the models the appropriate binary fraction and associated mass ratios and orbital characteristics.

The presence of binaries has a number of implications for Gaia. Astrometric measurements will be affected by orbital motion, so that the astrometric solutions need to include the simultaneous derivation of the binary parameters, as for Volume 10 of the Hipparcos catalog (Perryman et al. 1997). In many cases the signature of binarity will be subtle, for example in close systems, or very wide systems or where mass ratios are large; in some cases the binary will contain variable stars, which could result in photocentre changes, and thus errors in the parallax determination (Pourbaix, these proceedings). On the other hand, the combination of astrometric, spectroscopic and photometric instruments on Gaia will be orders of magnitude more powerful than any current facilities in detecting binary systems, and in determining their characteristics.

Fig. 6 shows how the whole range of orbital periods is covered with Gaia. Close pairs with periods from less than a day are detected in the RVS, while wide pairs with periods up to $\sim 10^{6} \mathrm{yr}$ are detected from astrometric motions. Binaries with periods from a few days to $\sim 5$ yr are detected with both techniques.

Spectroscopic double-lined eclipsing binaries will be most important for constraining stellar parameters. Currently only a few dozen of these are known, but $>100000$ are 


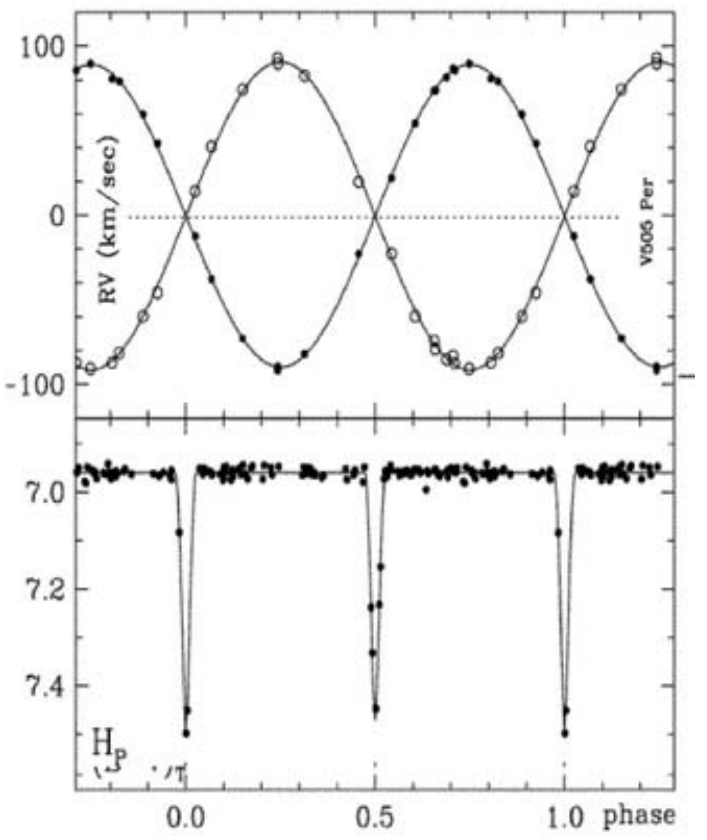

Figure 7. V505 Per radial velocities (top) and Hipparcos photometry (bottom) for this double-lined eclipsing binary. The data have been folded in phase. From Munari et al. (2001).

expected to be discovered for $V<15$, mostly with the RVS. Fig. 7 shows an example of ground-based spectroscopic observations of V505 Per, found to be eclipsing from Hipparcos photometry. Gaia will also provide astrometric measurements. Complete solutions will be obtained for masses, radii, luminosities, and semi-major axes. Some $1 \%$ of the sample, > 1000 binaries, will have exceptionally precise measurements and will form a reference sample for stellar parameter determination.

\subsection{Non-normal Stars}

A significant fraction of the Gaia sample will be of "peculiar" stars of various types. These continue to be very important in elucidating particular aspects of astrophysics and include stars with coronal emission, winds, accreting systems, magnetically interacting systems, eruptive, pulsating and oscillating systems, systems in particular stages of evolution (some very short), and so on. Even for relatively rare systems, the sample sizes of such systems will be transformed by Gaia.

This group is too diverse to provide anything more than a few examples in the RVS spectral range. Fig. 8 (left) shows examples of Carbon star spectra, in which the Ca triplet is completely dominated by other absorptions. Fig. 8 (right) shows spectra of halo symbiotics, in which subtle signatures of wind emission are evident - note the relative velocity shifts between these halo objects.

Fig. 9 shows spectra of nova-like Cataclysmic Variables in the RVS spectral range. Broad Paschen lines in emission are evident from the accretion disk, together with narrower components from the heated secondary star. The variation of these lines with orbital period allows the velocity structure and hence tidal effects in the disk to be determined; the multi-epoch nature of the Gaia survey is an important ingredient in this case. 

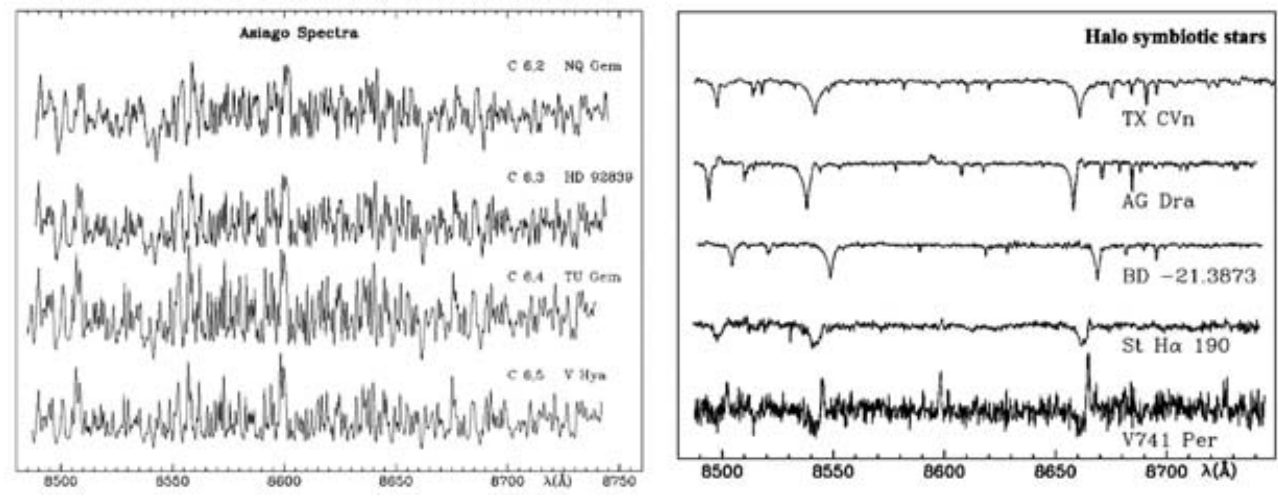

Figure 8. Left: Spectra of four Carbon stars in the RVS spectral range. Right: Halo symbiotic stars, some showing P Cygni profiles as a signature of wind emission in those systems. Both figures from Munari (2003b)

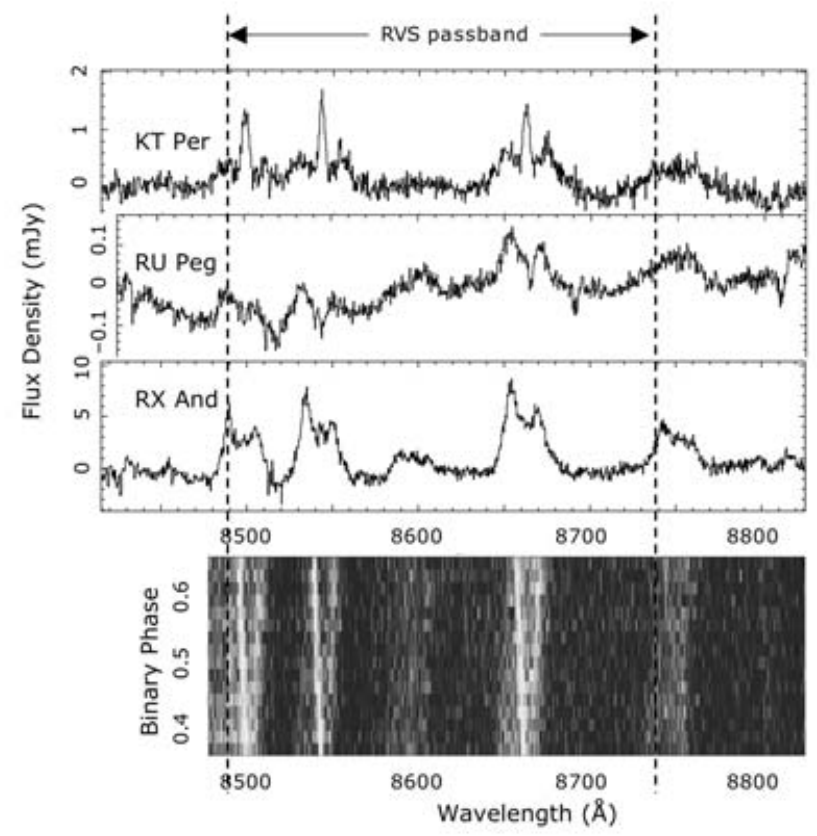

Figure 9. Spectra of the nova-like Cataclysmic Variables KT Per, RU Peg and RX And in the RVS spectral range. The plot at the bottom shows the variation of the spectral features as a function of time in the case of RX And. From Cropper \& Marsh 2003.

\section{RVS Characteristics}

RVS consists of an Offner-type spectrograph fed by a specific telescope on Gaia. The telescope also provides images for the Medium Band Photometer (see Katz et al. 2004). This optical system provides good image quality with minimal distortion over the field of view. Gaia scans the sky continuously, so images drift over the focal plane, with the detector clocking synchronised to the scan rate. Distortion is important to minimise in a scanning instrument, since it translates directly to image degradation. 


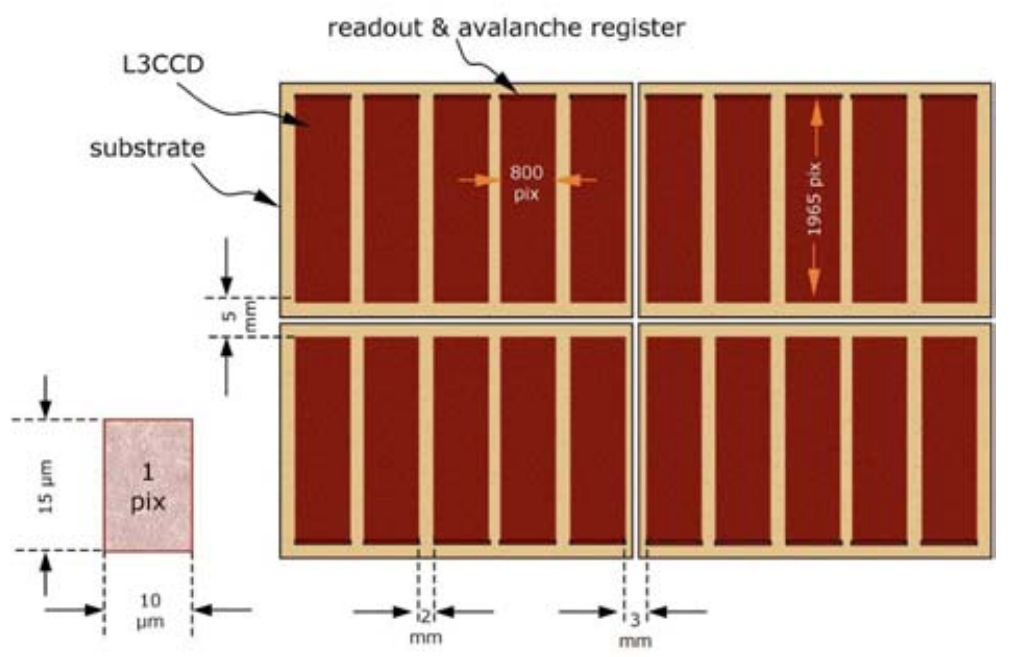

Figure 10. The layout of the RVS focal plane. This consists of 4 sets of 5 L3CCDs, with each set on a single substrate. The avalanche registers providing the gain in the devices follow the readout registers, and are located at the top and bottom of the focal plane.

The RVS focal plane consists of 20 CCDs of dimension $1965 \times 800$. The pixels are rectangular, with dimensions $10 \times 15 \mu \mathrm{m}$ - see Fig. 10. The CCDs are of a particular type, L3CCDs (low-light-level CCDs) which use avalanche gain in a multiplication register to amplify the signal before it is read out (Jerram et al. 2001). This effectively reduces the readout noise in the device, at the cost of some additional statistical noise but which does not dominate for fainter signals. The use of a number of CCDs allows the images to be remapped in software to correct for optical distortion, and the across-scan motion of the spectra (perpendicular to the scan and dispersion direction) to be corrected before the data from the individual CCDs are added together for telemetering.

RVS is a slitless spectrograph. The data format will be dispersed spectra from CCD detectors with spatial width $\sim 2$ pixels and spectral extension over $\sim 690$ pixels, set by the resolution requirement and the spectral bandpass. Radial velocities are then obtained by cross-correlation with a template. Most stars are detected at the limits of visibility and will require co-adding of all $\sim 100$ epochs of exposure scans. A simulation of the images from the RVS is shown in Fig. 11.

\section{Acknowledgements}

We are grateful to ESA for partially funding this work; also to the national funding agencies in the UK, France, Italy and Slovenia. We acknowledge the fruitful interactions with the Alenia/Alcatel and Astrium industry teams.

\section{References}

Binney, J. J., Dehnen, W., Houk, N., Murray, C. A., Penston, M. J. 1997, Proceedings of the ESA Symposium 'Hipparcos - Venice 97' ESA SP-402, p473

Blaauw, A. 1988, Proc. Colloq. Sitges, Hipparcos Scientific Aspects of the Input Catalogue Preparation II ed. Torra J., Turon C. p490. Comissió Interdepartamental de Recerca i Innovació Tecnològica, CIRIT, Generalitat de Catalunya, Barcelona

Boschi, F., Munari, U., Sordo, R., Marrese, P. M., 2003, In Symbiotic Stars Probing Stellar Evolution ed. R. L. M. Corradi, R. Mikolajewska \& T. J. Mahoney. ASP Conference Proceedings, vol. 303, p535. Astronomical Society of the Pacific. 


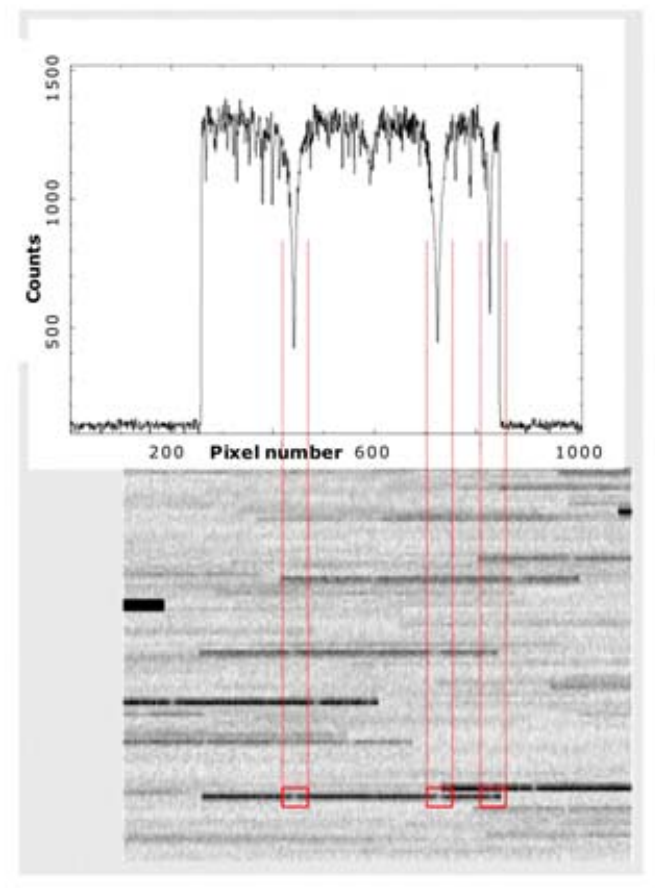

Figure 11. Bottom: a small section of simulated data from the RVS. The Ca absorption lines can be seen in the dispersed spectra. Top: an example of a spectrum extracted from the 2-dimensional data. The edges of the instrument wavelength bandpass are artificially sharp in this simulation.

Cropper, M., Marsh, T. 2003, In Gaia Spectroscopy: Science and Technology ed. U. Munari. ASP Conference Proceedings, vol. 298, p407. Astronomical Society of the Pacific.

Duchêne, G. 1999, A\&\&A 341, 547

Eggen, O. J., Lynden-Bell, D., Sandage, A. R. 1962, ApJ 136, 748

Favata, F., Perryman, M. A. C. 1997, In Proceedings of the ESA Symposium 'Hipparcos - Venice 97' ESA SP-402, p.771

Hardfing, P., Morrison, H. L., Olszewski, E. W., Arabadjis, J., Mateo, M., Dohm-Palmer, R. C., Freeman, K. C., Norris, J. E. 2001, (AJ 122, 1397

Helmi, A., de Zeeuw, P. T., 2000, MNRAS 319, 657

Helmi, A., White, S. D. M. 1999, MNRAS 307, 495

Ibata, R., Gilmore G., Irwin, M. 1994, Nature 370, 194

Jerram, P., Pool, P., Bell, R., Burt, D. J., Bowring, S., Spencer, S., Hazelwood, M., Moody, I., Catlett, N., Heyes, P. S. 2001, SPIE 4306, 178

Johnston, K. V., Hernquist, L., Bolte, M. 1996, ApJ 465, 278

Kapteyn, J. C. 1922, ApJ 55, 302

Katz, D., Munari, U., Cropper, M., and 47 co-authors 2004, MNRAS, in press

Munari, U. 2003a, In Gaia Spectroscopy: Science and Technology ed. U. Munari. ASP Conference Proceedings, vol. 298, p51. Astronomical Society of the Pacific.

Munari, U. 2003b, In Gaia Spectroscopy: Science and Technology ed. U. Munari. ASP Conference Proceedings, vol. 298, p227. Astronomical Society of the Pacific.

Munari, U., Tomov, T., Zwitter, T., Milone, E. F., Kallrath, J., Marrese, P. M., Boschi, F., Prsa, A., Tomasella, L., Moro, D. 2001, A\&A 378, 477

Navarro, J. F., Helmi, A., Freeman, K. C., 2004, ApJ 601, L43

Nordström, B., Mayor, M., Andersen, J., Holmberg, J., Pont, F., Jørgensen, B. R., Olsen, E. H., Udry, S., Mowlavi, N. 2004, AछA 418, 989 
Perryman, M. A. C. 1997, In Proceedings of the ESA Symposium 'Hipparcos - Venice 97' ESA SP-402

Perryman, M. A. C., Lindegren, L., Kovalevsky, J. and 16 coauthors 1997, A $\& A$ 323, L49

Perryman, M. A. C., deBoer, K. S., Gilmore, G., Høg, E., Lattanzi, M. G., Lindegren, L., Luri, X., Mignard, F., Pace, O., deZeeuw, P. T. 2001, A $\mathscr{E} A$ 369, 339

Quinn, P. J., Hernquist, L., Fullagar, D. P. 1993, ApJ 403, 74

Söderheljm, S., 2003, In Gaia Spectroscopy: Science and Technology ed. U. Munari. ASP Conference Proceedings, vol. 298, p351. Astronomical Society of the Pacific.

Soria, R., Cropper, M., Pakull, M., Mushotzky, R., Wu, K. 2004, astro-ph/0409568

Wilkinson, M. I., Vallenari, A., Turon, C. and 35 co-authors 2004, MNRAS submitted

\section{Discussion}

Dave Monet: Is the spectral range $8500 \AA$ to $8800 \AA$ with a resolution of about $1.5 \AA$ ?

MARK CRopper: The resolving power is 11000 and it's over a 25-nm range at $850 \mathrm{~nm}$. This range has also been subsequently adopted for the RAVE programme.

John Southworth: If you are getting velocities to $1 \mathrm{~km} \mathrm{~s}^{-1}$, you will need a good wavelength calibration. How do you do this?

MArk Cropper: You do. There are radial velocity standards, and Gaia is continually sweeping across these. There are not very many, but you are able to go to the primary references. The CORAVEL velocities are very good, so they are coming in to this range. But we've realised that the very best way for us to do this is through asteroids. You know the position very accurately, and you know the solar spectrum, so you can predict the radial velocity zero-point from asteroids to $\mathrm{ms}^{-1}$. That easily good enough for reference and there are plenty of them - we'll see several a day and that keeps everything calibrated. 